\title{
Mejoramiento de las Capacidades Tecnológicas en los Sectores Rurales de la Provincia de Imbabura
}

\section{Enhancing of Technological Capabilities in Rural Areas of the Province of Imbabura}

\author{
Stefany Flores Armas, Paul Rosero Montalvo, Edgar Maya Olalla, Diego Peluffo \\ Órdoñez \\ Universidad Técnica del Norte \\ Ibarra, Ecuador \\ Av. 17 de Julio 5-21 \\ \{scflores,pdrosero, dhpeluffo, eamaya\}@tun.edu.ec
}

\section{RESUMEN}

La Universidad Técnica del Norte a través de la carrera de ingeniería en Electrónica y Redes de Comunicación, fortaleciendo al proceso de vinculación con la sociedad, crea un proyecto que beneficia a la población de las zonas rurales de la provincia de Imbabura, y que, particularmente, tiene el objetivo de disminuir la brecha digital en colegios, brindado acceso a las tecnologías de información y comunicación. Para poner en acción el proyecto de mejoramiento de las capacidades tecnológicas, se consideró como población de interés a los individuos mayores de 12 años, hombres y mujeres, de diferentes niveles sociales, culturales y étnicos. Metodológicamente, se plantea una planificación de programas de capacitación en base a un diagnóstico de las necesidades, que posteriormente fueron evaluados con el propósito de reducir las deficiencias tecnológicas de las personas y así mejorar la calidad de vida con miras a reducir el analfabetismo digital. Dentro de la ejecución del proyecto, se toma como estrategia los Infocentros Comunitarios y propios laboratorios de computación de las instituciones educativas, los mismos que son punto de referencia para llegar a las comunidades, logrando capacitar en herramientas de ofimática básica, asistencias de redes de comunicación, Internet, mantenimiento de computadoras, y administración de páginas web. Posteriormente a la capacitación, claramente se pudo observar que, en los sectores de acción, satisficieron sus expectativas, adquirieron habilidades y destrezas de temas tecnológicos. En este artículo se presenta los aspectos metodológicos y resultados más importantes del proyecto.

Palabras Claves: Vinculación, universidad-sociedad, extensión universitaria, capacidades tecnológicas, sectores rurales 


\begin{abstract}
The engineering degree program in Electronics and Communication Networks from Universidad Técnica del Norte, with the aim of strengthening the society service processes, has created a project that benefits the population of the rural areas of the province of Imbabura. Such a project has as a main goal to reduce the digital divide in high schools by providing access to information and communication technologies to Imabura's rural population. To execute the improving technological capabilities project, it is considered as a population of interest, individuals (men and women) older than 12 from different social levels, cultures and ethnic backgrounds. Methodologically, training programs based on a needs' assessment are formulated, which are subsequently evaluated with the aim of reducing the digital illiteracy level in the population of interest. In turn, improving its technological deficiencies and its quality of life. Strategically, the Community Infocentros and school labs are considered, since they are reference points for reaching communities. Along with them, project executors are able to making training on basic office tools, basics on communication networks, Internet, computer maintenance, and men and women management of web pages. Following the training, it was observed that, in the fields of action, participants met their expectations, abilities and skills acquired technology issues. In this paper, the methodological aspects and main results of the project are presented.
\end{abstract}

KEYWORDS Society service, society-university, university extension, technological capabilities, rural areas.

\title{
INTRODUCCIÓN
}

La transferencia de tecnología es la transmisión del saber hacer, conocimientos científicos y tecnológicos hacia personas sin estas competencias con el fin de mejorar su calidad de vida generando riqueza social (Becerra, 2004), permite reducir brechas entre sectores poblacionales mediante buenas prácticas de uso para impulsar el desarrollo y capacitación de las personas e incrementar el interés en actividades de investigación y formación académica bajo un modelo lineal, cumpliendo fases y metas en las zonas de impacto sin fines comerciales (Del Socorro, Mejía, \& Schmal, 2006). La Universidad Técnica del Norte preocupada por el desarrollo de la comunidad ha fortalecido el proceso de vinculación con proyectos de transferencia de tecnología buscando generar mayor difusión de las comunicaciones, mejorar niveles educativos con un adelanto tecnológico adecuado (Yopo, 1975), (Universidad Técnica del Norte, 2015).

El proceso de uso tecnológico en Ecuador está avanzado, el término de analfabeto digital estadísticamente se está reduciendo, (INEC, 2015) indica que el $30 \%$ de las personas tiene este inconveniente, el proceso para evadirlo se lo 
realiza naturalmente en las zonas urbanas con la interacción de la sociedad, en el sector rural solo el $9 \%$ tiene acceso a una computadora en sus hogares, el acceso a tecnologías el sector urbano duplica a este sector, personas de edades superiores a 35 años no superan el $30 \%$ de acceso a internet y que posean computadora en sus hogares, teniendo en cuenta que los niños y jóvenes tienen un acceso del $60 \%$, da como resultado que los padres de familia tengan menor acceso a computadores, ocasionando problemas en la enseñanza sobre su correcto uso y el debido control parental de contenidos, como se muestra en la figura 1.

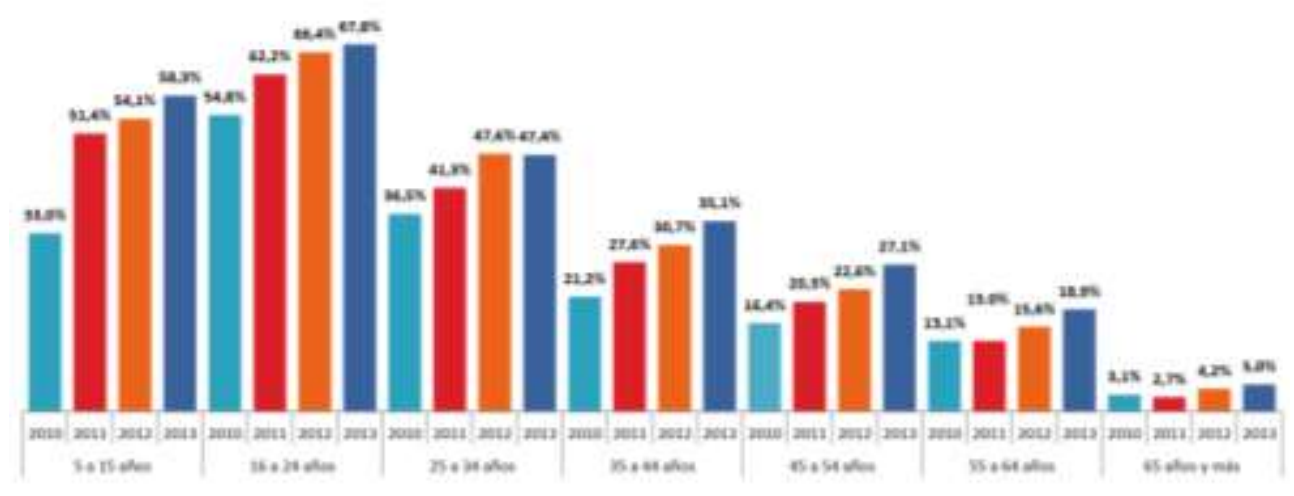

Figura 1 Porcentaje de personas que utilizan computadora por grupos de edad a nivel nacional según (INEC, 2015)

En la provincia de Imbabura según los datos (ECUACIFRAS, 2015) cuenta con un $30,9 \%$ de analfabetismo digital, con una población total de 398.244, donde su mayor incidencia es en mujeres y en el sector rural, 190.202 personas usaron un dispositivo móvil, 96.020 internet y 114.340 una computadora, es decir que solo el $36.4 \%$ de toda la población tiene acceso a un computador como se muestra en la figura 2.

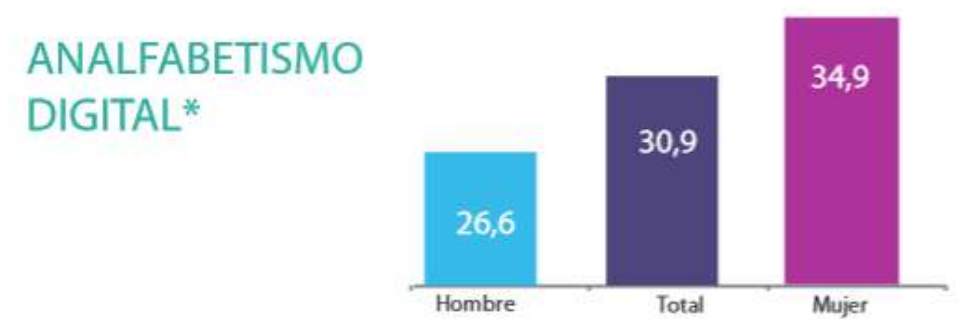

Figura 2 Tecnologías de la Información en Imbabura (ECUACIFRAS, 2015). 
Los jóvenes tienen la oportunidad de acceso al internet, la información encontrada se puede convertir en un distractor en clases y expone a contenidos sexuales a menores de edad; al momento de definir las amenazas más preocupantes, los padres identificaron a la pornografía en primer lugar (89,5\%), secundada por la pedofilia $(86,8 \%)$ y la persuasión de un adulto hacia un niño para que realice actividades sexuales, con el $75,7 \%$. El uso de tecnología en el sistema educativo ayuda al estudiante para ampliar sus conocimientos y tener una panorámica local, nacional, regional y mundial, para ello es necesario insertar a este proceso a todos quienes forman parte de nuestra sociedad: niños, jóvenes, padres y profesores deben permanecer en el proceso de aprendizaje continuo para canalizar las habilidades de los niños a un mejor bienestar y por ende la sociedad.

\section{MÉTODO}

El modelo planteado es lineal de transferencia de tecnología, que debe cumplir fases que se indica en el diagrama de bloques de la figura 3.

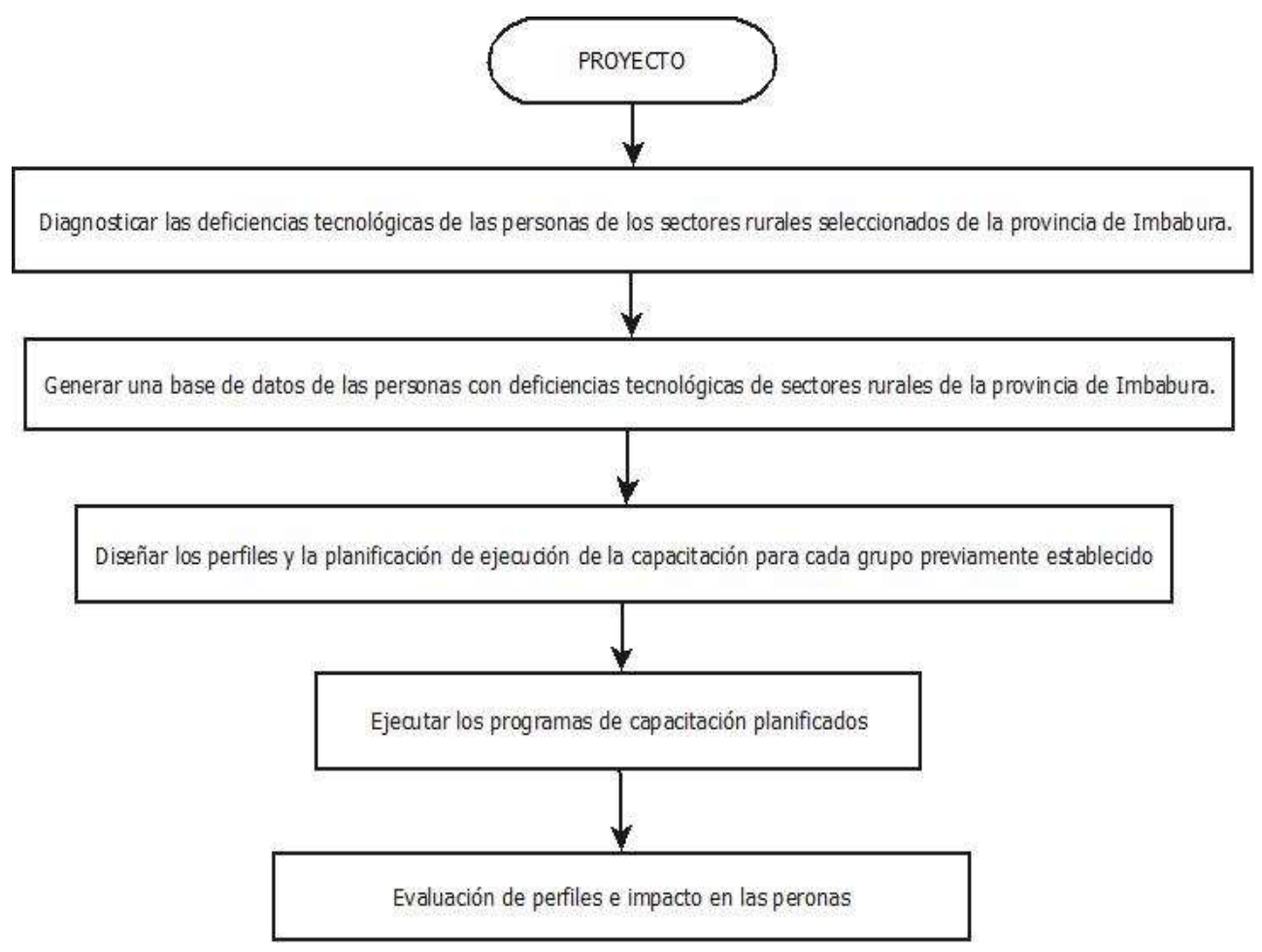

Figura 3 Fases del proyecto (Autoría) 
Los encargados del proceso son 40 estudiantes de $7 \mathrm{mo}$ a 10 mo semestre de la carrera de Electrónica y Redes de Comunicación matriculados legalmente en horas de vinculación, los estudiantes deben cumplir un total de 160 horas comprendidas en dos semestres, trabajan en grupos de 5 estudiantes que presentan una planificación e informe mensual y un informe final de acuerdo con las fases del proyecto planteado.

Todos los grupos de estudiantes cuentan con un docente tutor que monitorea y asiste a los estudiantes en todo el proceso, además es el encargado de generar los vínculos institucionales con la Universidad Técnica del Norte.

Las evaluaciones de deficiencias tecnológicas se establecieron por cantones de la provincia de Imbabura según los volúmenes de estudiantes y poder llegar a la mayor cantidad de beneficiados para apreciar el impacto de mejor manera. El Ministerio de Educación cuenta con un listado por zonas sobre la información de instituciones educativas, en la zona 1 del Ecuador como se muestra en la figura 4 se observa las diferentes instituciones académicas en relación al número de estudiantes y su modalidad, se establece como plan piloto realizar el proyecto solo en la provincia de Imbabura por la facilidad de acceso para los estudiantes.

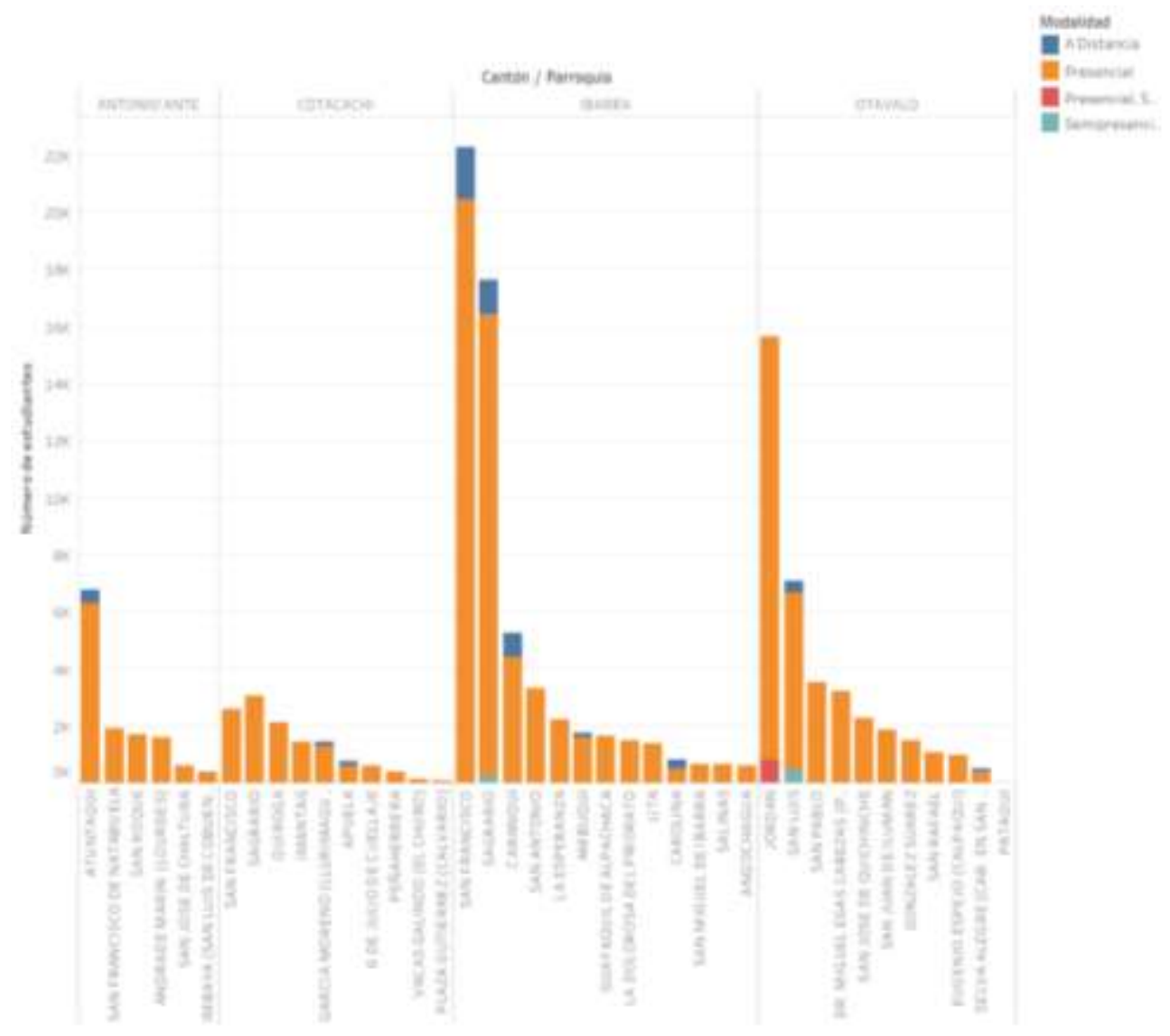

Figura 4 Información sobre número de estudiantes según cantones de la provincia de Imbabura, (Autoría). 
Las encuestas realizadas vía online a los jóvenes y padres de familia en instituciones con mayor número de estudiantes en los diferentes cantones de la provincia, utilizando el principio de Pareto de determinar con el $20 \%$ de estudiantado las tendencias del restante $80 \%$. Los resultados de le encuesta en las instituciones educativas de la provincia de Imbabura indicaron las deficiencias en herramientas de ofimática básica, asistencias de redes de comunicación, Internet, mantenimiento de computadoras, y administración de páginas web. En la figura 5 se muestra las instituciones educativas con mayor número de estudiantes en relación al número de profesores.
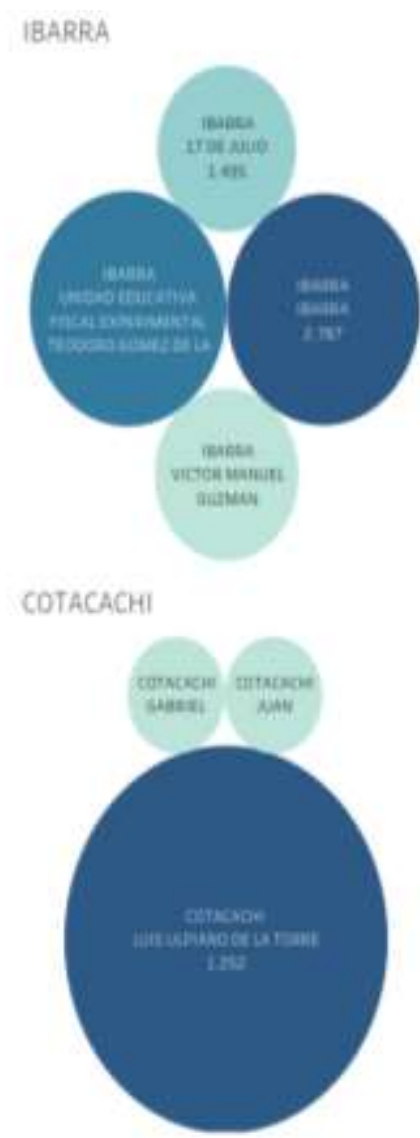

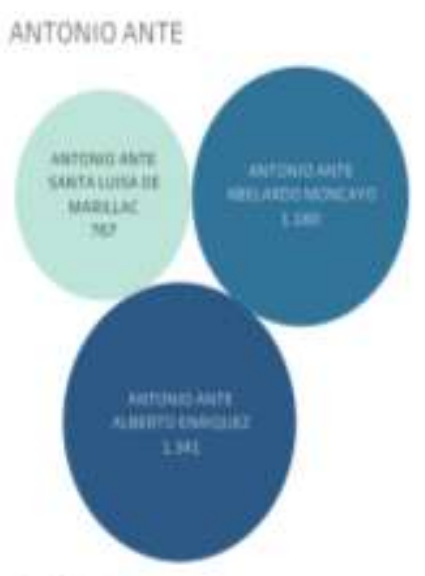

OTAVALO

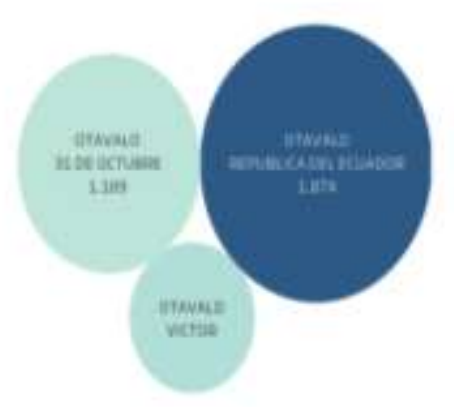

Figura 5 Ranking por número de estudiantes en los cantones de la provincia de Imbabura, (Autoría).

Mediante una alianza estratégica para ciertas instituciones que no contaban con un centro de cómputo adecuado, la Corporación Nacional de Telecomunicaciones facilitó el uso de los infocentros de la provincia para acceder a las encuestas y cursos, no se toma en cuenta los cantones de Urcuquí y Pimampiro por el bajo número de estudiantes y las dificultades de llegar a estos lugares. 
Con las deficiencias en los estudiantes se diseñaron los perfiles como lo indica en la figura 6, además de generar una charla a los padres de familias sobre la importancia de la tecnología en la educación y en el desarrollo del país, conjuntamente con herramientas para controlar el acceso a contenidos desde los colegios y los hogares.

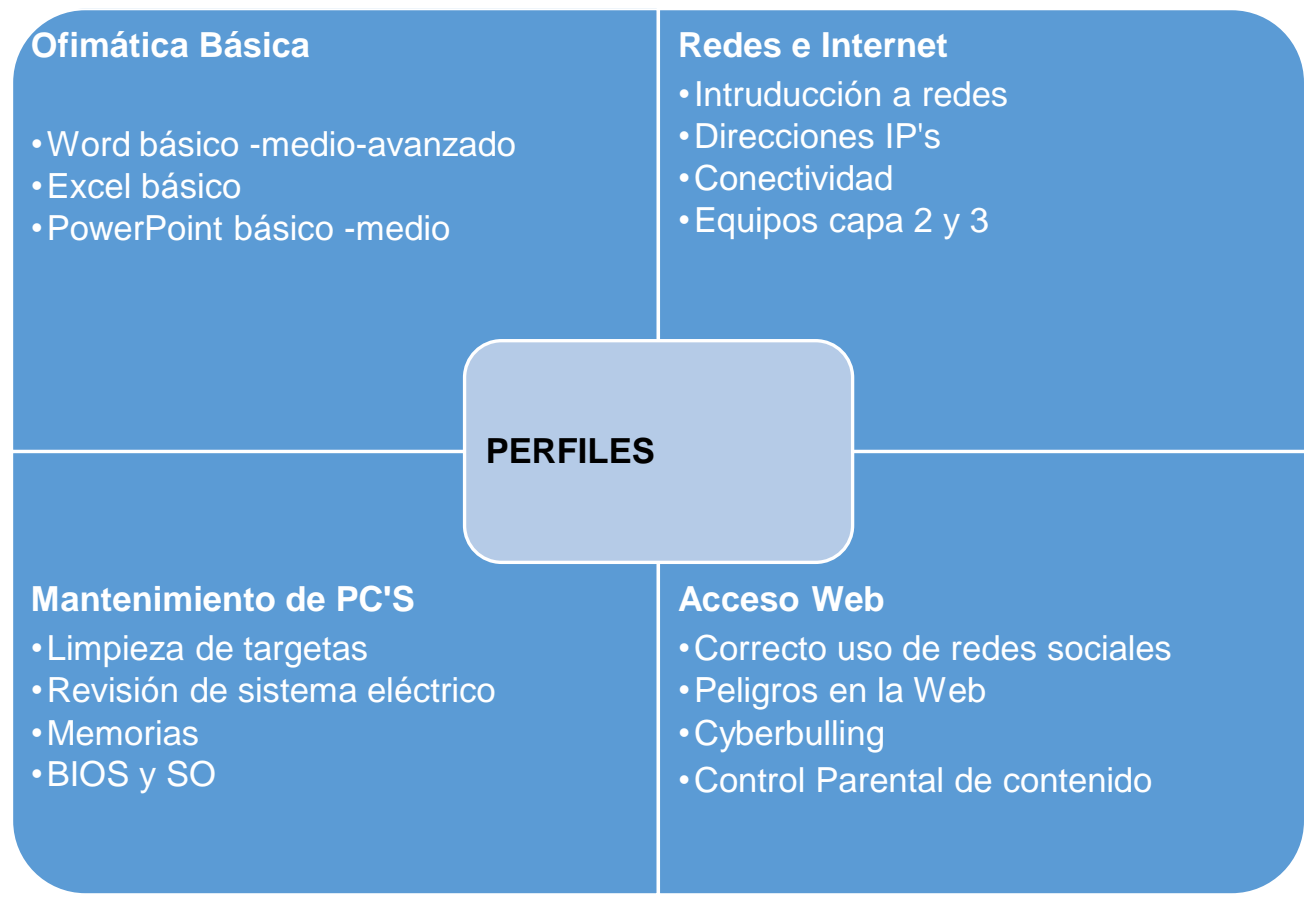

Figura 6 Perfiles para transferencia de tecnología en la provincia de Imbabura, (Autoría).

El despliegue de la transferencia de tecnología con las capacitaciones en relación a los perfiles determinados se filtraron todas las instituciones educativas que se encuentren en la zona rural de Imbabura, que tengan horario matutino para coordinar con los estudiantes de la carrera de Electrónica y Redes de Comunicación y puedan trasladarse sin problemas. Con un recurso de 40 estudiantes se dividieron en 8 grupos de 5 personas, donde cada uno de ellos es el encargado de trabajar con una institución educativa, el curso a trabajar es según los criterios de las autoridades de cada unidad y que todos los horarios de los involucrados concuerden con una planificación de 9 meses, es decir sin contar con los adolescentes de segundo y tercero de bachillerato. Al desplegar los grupos de estudiantes se asignaron 2 grupos por cantón donde se cuente con el mayor número de estudiantes con el acuerdo que los jóvenes capacitados puedan replicar dichos cursos en su misma institución o unidades de menor tamaño con la asesoría de los estudiantes de ingeniería como se muestra en la tabla 1. 
Tabla 1 Unidades educativas beneficiadas de transferencia tecnológica, (Autoría).

\begin{tabular}{|c|c|c|c|c|}
\hline CANTÓN & $\begin{array}{l}\text { UNIDAD } \\
\text { EDUCATIVA }\end{array}$ & $\begin{array}{l}\text { \# } \\
\text { ESTUDIANTES }\end{array}$ & $\begin{array}{l}\text { \# } \\
\text { PROFESORES }\end{array}$ & CURSO \\
\hline \multirow[t]{2}{*}{$\begin{array}{l}\text { ANTONIO } \\
\text { ANTE }\end{array}$} & $\begin{array}{l}\text { ANTONIO } \\
\text { ANTE }\end{array}$ & 402 & 19 & $\begin{array}{l}25 \\
\text { Estudiantes } \\
\text { de } 1 \\
\text { bachillerato }\end{array}$ \\
\hline & $\begin{array}{l}\text { ABELARDO } \\
\text { MONCAYO }\end{array}$ & 1160 & 53 & $\begin{array}{l}30 \\
\text { Estudiantes } \\
\text { de } 1 \\
\text { bachillerato }\end{array}$ \\
\hline \multirow[t]{2}{*}{ COTACACHI } & $\begin{array}{c}\text { LUIS } \\
\text { PLUTARCO } \\
\text { CEVALLOS }\end{array}$ & 435 & 21 & $\begin{array}{l}31 \\
\text { Estudiantes } \\
\text { de } 10 \text { mo } \\
\text { año }\end{array}$ \\
\hline & $\begin{array}{c}\text { RED } \\
\text { EDUCATIVA } \\
\text { CUELLAJE- } \\
\text { ESCUELA } \\
\text { CESAR } \\
\text { BORJA }\end{array}$ & 339 & 21 & $\begin{array}{l}23 \\
\text { Estudiantes } \\
\text { de } 10 \text { mo } \\
\text { Estudiantes } \\
\text { de } 1 \\
\text { bachillerato }\end{array}$ \\
\hline \multirow[t]{2}{*}{ IBARRA } & $\begin{array}{l}\text { VALLE DEL } \\
\text { CHOTA }\end{array}$ & 722 & 42 & $\begin{array}{l}28 \\
\text { Estudiantes } \\
\text { de } 1 \\
\text { bachillerato }\end{array}$ \\
\hline & RUMIPANBA & 308 & 17 & $\begin{array}{l}23 \\
\text { Estudiantes } \\
\text { de } 1 \\
\text { bachillerato }\end{array}$ \\
\hline \multirow[t]{2}{*}{ OTAVALO } & $\begin{array}{c}\text { CESAR } \\
\text { ANTONIO } \\
\text { MOSQUERA }\end{array}$ & 709 & 28 & $\begin{array}{l}33 \\
\text { Estudiantes } \\
\text { de } 9 \text { año }\end{array}$ \\
\hline & $\begin{array}{l}\text { CARLOS } \\
\text { UBIDIA } \\
\text { ALBUJA }\end{array}$ & 501 & 23 & $\begin{array}{l}28 \\
\text { Estudiantes } \\
\text { de } 1 \\
\text { bachillerato }\end{array}$ \\
\hline
\end{tabular}

El costo de los materiales para la capacitación de los estudiantes y padres de familia se detallan en la tabla 2. 
Tabla 2 Costo de materiales para la entrega de guías, (Autoría)

\begin{tabular}{llll}
\hline Cant. & Descripción & Valor Unidad & Total \\
\hline $\mathbf{1 0 0}$ & Impresión de guías & 5.25 & 525 \\
\hline $\mathbf{1}$ & Trasporte & 50 & 50 \\
\hline $\mathbf{1}$ & Material de oficina & 50 & 50 \\
\hline $\mathbf{1}$ & Refrigerios & 100 & 100 \\
\hline & & TOTAL & $\$ 725$ \\
\hline
\end{tabular}

Hay que indicar que en los costos no se toman en cuenta las horas de trabajo de los estudiantes y profesores por ser parte de un proceso de formación estudiantil, además de parte del departamento de vinculación de la Universidad Técnica del Norte se cuentan con equipos como proyectores, computadores, etc. sin costo para los beneficiados.

\section{RESULTADOS}

Una vez concluido el proceso de transferencia tecnológica se tuvo a 221 estudiantes capacitados con certificados de 40 horas en cursos con las diferentes temáticas planteadas, en cada institución se acordó que los estudiantes agentes de cambio en su trabajo de monografía para su bachillerato tengan la facilidad de transferir sus conocimientos a otras instituciones cercanas o cursos del mismo establecimiento para aumentar el número de beneficiarios, este proceso sigue siendo monitoreado por los siguientes estudiantes que trabajan en vinculación.

Una vez concluido el proceso se cuenta con material y evaluaciones de cada perfil y validados con los mismos estudiantes, se realizaron charlas de los estudiantes hacia los padres de familia para ampliar el impacto de sus conocimientos.

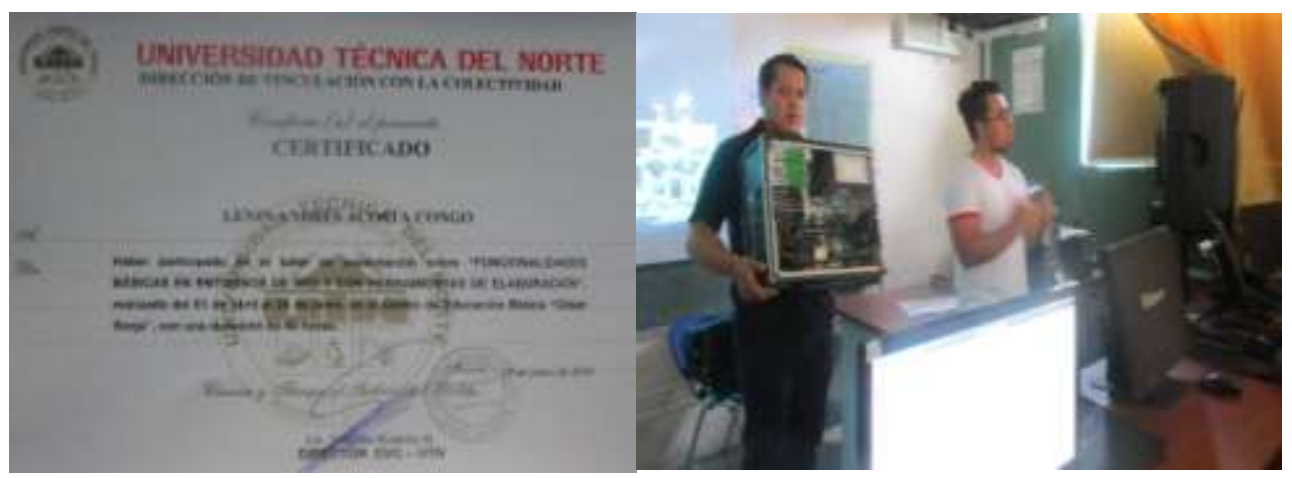




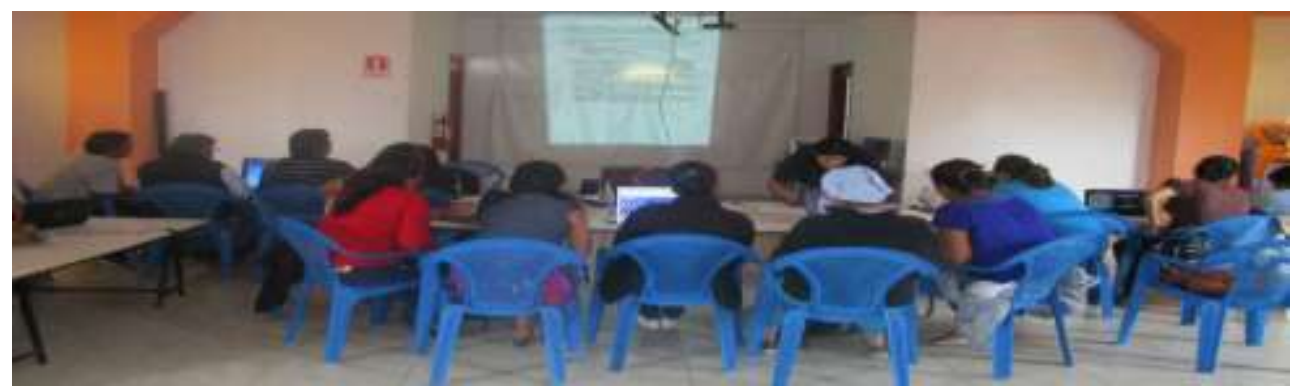

Figura 7 Estudiantes de ingeniería capacitando a estudiantes de colegio y padres de familia.

Con 221 estudiantes replicando dichos cursos a un promedio de 20 personas, se tendrá un alcance de 4420 personas directamente beneficiados, haciendo relación que una familia tiene 4 personas (INEC, 2015), en total se contará con 17680 personas que conocerán estos nuevos procesos y conocimientos tecnológicos. El índice de analfabetismo digital era de un 30,9\% con una población de 398.244, es decir 155315 personas, al finalizar el proyecto con 17680 beneficiadas se reduce en un 11\% la brecha digital en los sectores rurales de la provincia de Imbabura en un año de trabajo, con el desarrollo de proyectos subsecuentes se espera reducir en el 2018 el $50 \%$ de personas analfabetas tecnológicas.

\section{REFERENCIAS}

Becerra, M. (2004). La transferencia de tecnología en Japón. Conceptos y enfoques. Cienca VII, Universidad Autónoma de Nuevlo León, 20-25.

Del Socorro, M., Mejía, J. C., \& Schmal, R. (2006). Un Acercamiento al Concepto de la Transferencia de Tecnología de las Universidad y sus Diferentes Manifestaciones. PANORAMA SOCIOECONÓMICO, 70-81.

ECUACIFRAS. (20 de Febrero de 2010). Obtenido de ECUACIFRAS: http://www.ecuadorencifras.gob.ec/wp-content/descargas/Manu-lateral/Resultadosprovinciales/imbabura.pdf

INEC. (2015). INEC. Obtenido de http://www.inec.gob.ec

Saki, Claudio. (2005). Transferencia de Tecnología en la educación en el área científica. Instituto de Física, Sao Paulo.

Lugo, T. (2015) Las Políticas TIC en América Latina: prioridad de las agendas educativas. Red Latinoamericana de Portales Educativos 
Borja Moreno (1984). «El eslabón perdido». http://www.itu.int/osg/spu/sfo/missinglink/index.html (en inglés). Maitland report. Consultado el 24/09/15.

Hoffman, D.L, Novak, T.P. y Schlosser, A. E. (2001) The evolution of the digital divide: Examining the relationship of race to internet access and usage over time. En Compaine, B. Digital Divide. Cambridge, Massachusetts: The MIT Press

Grosse, Robert (1996). «international Technology Transfer in Services». Journal of International Business Studies 27: 782.

Cohen, W.; Nelson, R.; Walsh, J. (2002). Links and impacts: the influence of public research on industrial R\&D. (48). pp. 1-23. 